\title{
Achados da videoendoscopia da deglutição em adultos com disfagia orofaríngea neurogênica
}

\author{
Findings of fiberoptic endoscopy of swallowing in adults with \\ neurogenic oropharyngeal dysphagia
}

\author{
Moisés Andrade dos Santos de Queiroz ${ }^{1}$, Renata Cavalcante Barbosa Haguette ${ }^{2}$, Erik Frota Haguette
}

\begin{abstract}
RESUMO
Objetivo: Descrever os principais achados da videoendoscopia da deglutição em adultos com disfagia orofaríngea neurogênica. Métodos: Foi realizado um estudo retrospectivo de 46 prontuários de pacientes com diagnóstico de disfagia orofaríngea neurogênica, atendidos no período de julho de 2004 a dezembro de 2007 no Ambulatório de Disfagia da Universidade de Fortaleza. A média de idade dos pacientes foi de 58,9 anos, variando entre 18 e 85 anos, sendo 51,1\% (22/43) do gênero masculino e 48,9\% (21/43) do gênero feminino. Os exames foram considerados alterados na presença de uma ou mais das seguintes manifestações disfágicas: escape precoce posterior, atraso na deglutição, estase em valéculas e/ou recessos piriformes, penetração laríngea e/ou aspiração traqueal. Resultados: Os principais achados observados foram, em ordem decrescente de ocorrência: estase em valéculas após a ingestão da consistência pastosa grossa, atraso na deglutição durante a deglutição de alimentos nas consistências pastosa grossa e sólida, presença de estase em recessos piriformes após a deglutição da consistência pastosa fina, escape posterior de líquido, penetração laríngea e aspiração traqueal de líquidos antes da deglutição. Conclusão: As principais manifestações disfágicas observadas estiveram relacionadas às alterações das fases voluntárias da deglutição e ao aumento das consistências dos alimentos. A videoendoscopia da deglutição demonstrou ser um instrumento eficaz no diagnóstico das disfagias orofaríngeas.
\end{abstract}

Descritores: Deglutição; Disfagia; Endoscopia/métodos; Endoscopia/utilização; Transtornos de deglutição

\section{INTRODUÇÃO}

A deglutição é um processo dinâmico e intermitente realizado por um conjunto de mecanismos neuromotores, coordenados pelo córtex cerebral, tronco cerebral e nervos encefálicos ${ }^{(1)}$. Composta por fases intrinsecamente relacionadas e divididas, didaticamente, de acordo com a região em que se desenvolve em voluntárias (preparatória oral e oral) e involuntárias (faríngea e esofágica), exerce a função de conduzir o alimento da cavidade oral ao estômago de forma segura, por meio de uma coordenação precisa, mantendo o estado nutricional e protegendo a via aérea do indivíduo ${ }^{(1-2)}$.

Pesquisa do Programa de Bolsas de Iniciação Científica (PROBIC) realizada no Ambulatório de Disfagia do Núcleo de Atenção Médica Integrada (NAMI) da Universidade de Fortaleza - UNIFOR - Fortaleza (CE), Brasil.

(1) Acadêmico do Curso de Fonoaudiologia da Universidade de Fortaleza UNIFOR - Fortaleza (CE), Brasil.

(2) Mestre, Professora Assistente do Curso de Fonoaudiologia da Universidade de Fortaleza - UNIFOR - Fortaleza (CE), Brasil.

(3) Mestre, Professor Assistente do Curso de Fonoaudiologia da Universidade de Fortaleza - UNIFOR - Fortaleza (CE), Brasil.

Endereço para correspondência: Renata Cavalcante Barbosa Haguette. Av.

Dom Luís, 1233, sala 1301, Aldeota, Fortaleza (CE), Brasil, CEP: 60125-150.

E-mail: renatahaguette@unifor.br

Recebido em: 23/4/2008; Aceito em: 24/7/2009
Durante a fase preparatória oral, o alimento é organizado dentro da cavidade oral, por intermédio da movimentação da língua, da insalivação e da mastigação. Ao final desta fase, o bolo alimentar estará formado e será posicionado entre a língua e o palato duro para sua posterior propulsão em direção à faringe, caracterizando a fase oral propriamente dita da deglutição, que finaliza com o disparo do reflexo da deglutição ${ }^{(3)}$.

Durante a fase faríngea, ao disparo do reflexo da deglutição inicia-se a proteção das vias aéreas com o selamento da nasofaringe, a elevação do complexo hiolaríngeo, o fechamento das pregas vocais, sendo o mais importante para a proteção laríngea, e o abaixamento da epiglote. Os músculos constritores faríngeos contribuem para o transporte do bolo alimentar, por meio dos movimentos peristálticos associados à gravidade, ao músculo cricofaríngeo que, estando relaxado, permite a passagem do alimento para o esôfago ${ }^{(1,4)}$.

Os distúrbios da deglutição, especificamente as disfagias, são caracterizados por prejuízos no funcionamento de qualquer fase e/ou entre as fases da deglutição, em consequência de comprometimentos neurológico, mecânico ou psicogênico ${ }^{(2,5)}$.

As disfagias orofaríngeas estão frequentemente associadas ao quadro clínico de diversos distúrbios neurológicos, afetando, com maior repercussão, tanto a fase oral quanto a faríngea da dinâmica da deglutição ${ }^{(6)}$, alterando negativamente 
a qualidade de vida do indivíduo devido, principalmente, aos riscos à desnutrição, desidratação e infecções pulmonares por aspiração traqueal ${ }^{(7-8)}$.

Os métodos mais utilizados para o diagnóstico das disfagias são a avaliação clínica e os exames objetivos, como a videoendoscopia e a videofluoroscopia da deglutição ${ }^{(8-9)}$.

A videoendoscopia da deglutição (VED) apresenta resultados comparáveis à videofluoroscopia ${ }^{(10)}$, sendo um método de fácil realização, baixo custo, sem exposição à radiação, podendo ser realizado ao lado do leito do paciente; não oferece riscos ao indivíduo, na maioria dos casos, podendo ser repetido diversas vezes para a observação do sucesso da terapia fonoaudiológica ${ }^{(11)}$.

O exame consiste em uma nasofibrolaringoscopia tradicional com o oferecimento de alimentos tingidos com corantes, possibilitando uma visualização da dinâmica da deglutição, especialmente em sua fase faríngea, cuja grande importância está na presença do caminho concomitante entre as vias respiratória e digestiva ${ }^{(12)}$.

A realização da VED fornece subsídios para a implementação de estratégias compensatórias apropriadas, tais como: a determinação das consistências alimentares e das manobras facilitadoras a serem utilizadas, que contribuem para a redução e/ou eliminação do risco de penetração laríngea e/ou aspiração traqueal ${ }^{(13)}$.

O objetivo deste estudo foi descrever os principais achados da videoendoscopia da deglutição em adultos com diagnóstico de disfagia orofaríngea neurogênica.

\section{MÉTODOS}

Esta pesquisa foi avaliada e aprovada pelo Comitê de Ética em Pesquisa da Universidade de Fortaleza (UNIFOR) sob o número 193/2006.

Foi realizado um estudo retrospectivo em 46 prontuários de pacientes com diagnóstico de disfagia orofaríngea neurogênica, que realizaram avaliação clínica e videoendoscópica da deglutição, no período de julho de 2004 a dezembro de 2007, no Ambulatório de Disfagia do Núcleo de Atenção Médica Integrada (NAMI) da Universidade de Fortaleza (UNIFOR), na cidade de Fortaleza (CE).

A coleta dos dados foi realizada durante os meses de agosto a dezembro de 2007, onde foram selecionados todos os pacientes com diagnóstico de disfagia orofaríngea neurogênica acima de 18 anos.

Dentre os casos considerados elegíveis, foram excluídos aqueles que não apresentaram manifestações disfágicas durante a fase faríngea da deglutição, caracterizando uma amostra de 43 pacientes com o diagnóstico de disfagia orofaríngea neurogênica, submetidos à VED.

A média de idade dos pacientes foi de 58,9 anos, variando entre 18 e 85 anos, sendo $51,1 \%$ (22/43) do gênero masculino e $48,9 \%$ (21/43) do gênero feminino. A maioria (37,2\% - 16/43) dos pacientes apresentou diagnóstico médico de acidente vascular encefálico (AVE), 27,9\% (12/43) de doença neurológica a esclarecer, $6,9 \%$ (3/43) de traumatismo crânio encefálico (TCE) e 27,9\% (12/43) corresponderam a outras desordens neurológicas, como: doença de Parkinson, paralisia cerebral, miastenia gravis, paralisia bulbar progressiva, polimialgia traumática com atrofia muscular, trombose arterial basilar e miopatia mitocondrial, paralisia facial periférica, espondilodiscopatia degenerativa C5/C6, sequela de lesão cervical, paralisia de prega vocal, tumor de glomus jugular e transtorno degenerativo.

Os instrumentos empregados na coleta de dados consistiram dos protocolos de avaliação clínica (Anexo 1) e videoendoscópica (Anexo 2) da deglutição, utilizados no Ambulatório de Disfagia do NAMI - UNIFOR.

O nasofibroscópio utilizado foi o Olympus ${ }^{\circledR} \mathrm{P} 4$, acoplado à câmera de vídeo Wolf, ao videocassete Toshiba ${ }^{\circledR}$ e ao monitor Sony ${ }^{\circledR}$ colorido. Os exames foram gravados em fitas VHS.

A avaliação videoendoscópica da deglutição foi realizada e interpretada pelo médico otorrinolaringologista e pela fonoaudióloga do ambulatório. O paciente permaneceu na posição sentada e o nasofibroscópio foi introduzido pela narina, sem utilização de anestésico ou vasoconstritor tópico. Antes do início do estudo da deglutição foram observadas as fossas nasais e o fechamento velofaríngeo durante a fonação e a deglutição de saliva. $\mathrm{O}$ aparelho foi avançado através da rinofaringe em direção à orofaringe, observando-se a base da língua, a valéculas, os recessos piriformes, as paredes: laterais e posterior da faringe e as pregas vocais em repouso e durante a fonação.

Iniciou-se a avaliação funcional da deglutição com o oferecimento de alimentos nas consistências líquida, pastosa fina, pastosa grossa e sólida, tingidos com corante inorgânico azul para facilitar a sua visualização durante o exame. Os alimentos de consistência líquida foram oferecidos no copo (40 $\mathrm{ml})$, os pastosos finos e grossos na colher $(5 \mathrm{ml})$ e os sólidos diretamente na cavidade oral do paciente.

Alguns pacientes realizaram a VED apenas com a ingestão da saliva corada, devido à impossibilidade de alimentação por via oral destes, ou quando observada uma grande quantidade de estase salivar em valéculas e/ou recessos piriformes após três movimentos deglutitórios, o que é considerado um importante fator de risco para a penetração laríngea e/ou aspiração traqueal ${ }^{(10,14)}$.

Inicialmente, foi solicitado ao paciente que mantivesse o alimento na boca, avaliando a sua capacidade de contenção oral sob comando. Em seguida, o exame foi conduzido com a administração livre das demais consistências alimentares, de acordo com as possibilidades de cada paciente.

Neste estudo, em virtude da impossibilidade de visualizar a fase faríngea da deglutição, devido ao fechamento da musculatura faringolaríngea no momento da deglutição (efeito white out), classificamos a ocorrência destes sintomas, de acordo com a sua ocorrência antes ou após a deglutição.

O trânsito orofaríngeo foi avaliado de acordo com as seguintes variáveis: início da deglutição reflexa, clearance faríngeo e proteção das vias aéreas. A ocorrência do efeito white out foi utilizada para determinar o início da deglutição. Quanto ao clearance faríngeo analisou-se a presença ou ausência de estase alimentar em recessos faríngeos. Já, em relação à proteção das vias aéreas, investigou-se a ocorrência de adução glótica e tosse reflexa durante os episódios de penetração laríngea e/ ou aspiração traqueal, além do teste da sensibilidade laríngea conforme descrição a seguir. 
A sensibilidade laríngea foi testada, por meio do toque com a extremidade distal do aparelho nas pregas vocais, aritenóides e/ou bandas ventriculares, permitindo a observação da ocorrência de adução glótica e tosse reflexa.

Os exames foram considerados alterados na presença de uma ou mais das seguintes manifestações disfágicas: escape precoce do alimento para a hipofaringe, atraso no trânsito faríngeo da deglutição, acúmulo de resíduo alimentar em valéculas e/ou recessos piriformes após a deglutição, penetração laríngea e/ou aspiração traqueal e diminuição ou ausência da sensibilidade laríngea.

Realizamos uma análise descritiva a partir dos dados coletados, comparando os percentuais encontrados e identificando as consistências que apresentaram maior significância nos achados.

\section{RESULTADOS}

Dos pacientes que apresentaram o resultado de deglutição alterada durante a VED, 86\% (37/43) realizaram o exame com a ingestão de alimentos corados, enquanto $14 \%$ (6/43) foram avaliados somente com a ingestão de saliva corada.

As manifestações disfágicas mais relevantes observadas nos pacientes que realizaram a VED com apenas com a ingestão de saliva corada incluíram: penetração laríngea e estase salivar em valéculas e recessos piriformes (100\% - 6/6), atraso na deglutição $(83,3 \%$ - 5/6), escape posterior e aspiração traqueal $(66,7 \%$ - 4/6) e diminuição da sensibilidade laríngea $(33,3 \%$ - 2/6) dos casos (Tabela 1$)$.

Os principais achados observados na realização da VED com a administração dos alimentos corados foram, em ordem decrescente de ocorrência: estase em valéculas após a ingestão da consistência pastosa grossa $(62,2 \%$ - 23/43), atraso na deglutição durante a deglutição de alimentos nas consistências pastosa grossa e sólida $(54,1 \%$ - 20/43), presença de estase em recessos piriformes após a deglutição da consistência pastosa fina $(48,6 \%$ - 18/43), escape posterior de líquido $(43,2 \%$ 16/43), penetração laríngea (40,5\% - 15/43) e aspiração traqueal $(16,2 \%$ - 6/43) de líquidos antes da deglutição.

A Tabela 2 apresenta detalhadamente as principais manifestações observadas nos pacientes que realizaram a VED com a ingestão de alimentos corados, classificados segundo a consistência alimentar.
Tabela 1. Manifestações disfágicas observadas por meio da videoendoscopia da deglutição com a administração de saliva corada

\begin{tabular}{lcc}
\hline Manifestação disfágica & \multicolumn{2}{c}{ Saliva corada } \\
\cline { 2 - 3 } & $\mathrm{P}(\%)$ & $\mathrm{A}(\%)$ \\
\hline Escape posterior & $4(66,7)$ & $2(33,3)$ \\
Atraso na deglutição & $5(83,3)$ & $1(16,7)$ \\
Estase em valéculas & $6(100,0)$ & $0(0,0)$ \\
Estase em recessos piriformes & $6(100,0)$ & $0(0,0)$ \\
Penetração laríngea & $6(100,0)$ & $0(0,0)$ \\
Aspiração traqueal & $4(66,7)$ & $2(33,3)$ \\
\hline
\end{tabular}

Legenda: $\mathrm{P}=$ número de pacientes com presença de manifestação disfágica para saliva corada; $\mathrm{A}$ = número de pacientes com ausência de manifestação disfágica para saliva corada

Teste estatístico comparativo entre os percentuais

A diminuição da sensibilidade laríngea foi observada em apenas 16,2\% (6/37) dos pacientes que realizaram a VED com a ingestão de alimentos corados.

\section{DISCUSSÃO}

O escape precoce posterior está relacionado com o comprometimento motor da fase oral da deglutição, estruturas osteomúsculo-articulares responsáveis pela qualidade da ejeção, onde há o escape do alimento da cavidade oral para a faringe antes do disparo do reflexo da deglutição, o que pode resultar em uma aspiração traqueal, pois neste momento a via aérea encontra-se desprotegida.

A maior ocorrência do escape precoce posterior foi durante a administração da consistência líquida, presente em 43,2\% (16/43) dos sujeitos, sendo seguida pela pastosa fina em $31,1 \%$ (13/43), pastosa grossa em 18,9\% (7/43) e sólida em 8,1\% (3/43). Acredita-se que esta dificuldade para a contenção da consistência líquida seja decorrente do volume de difícil controle e da menor densidade e viscosidade desta, o que exige um maior controle oral ${ }^{(15)}$.

O atraso no disparo do reflexo da deglutição esteve igualmente comprometido para as consistências: pastosa grossa e sólida em 54,1\% (20/43), seguida da pastosa fina em 48,6\% (18/43) e a líquida em 35,1\% (13/43).

Observou-se piora da dificuldade para preparar, organizar, manipular e propulsionar o bolo alimentar, de acordo com o

Tabela 2. Manifestações disfágicas observadas por meio da videoendoscopia da deglutição com a ingestão de alimentos corados

\begin{tabular}{|c|c|c|c|c|c|c|c|c|}
\hline \multirow[t]{2}{*}{ Manifestação disfágica } & \multicolumn{2}{|c|}{ Líquida } & \multicolumn{2}{|c|}{ Pastosa fina } & \multicolumn{2}{|c|}{ Pastosa grossa } & \multicolumn{2}{|c|}{ Sólida } \\
\hline & $\mathrm{P}(\%)$ & $A(\%)$ & $\mathrm{P}(\%)$ & $A(\%)$ & $\mathrm{P}(\%)$ & $A(\%)$ & $\mathrm{P}(\%)$ & $A(\%)$ \\
\hline Escape posterior & $16(43,2)$ & $21(56,8)$ & $13(35,1)$ & $24(64,9)$ & $7(18,9)$ & $30(81,1)$ & $3(8,1)$ & $34(91,9)$ \\
\hline Atraso na deglutição & $13(35,1)$ & $24(64,9)$ & $18(48,6)$ & $19(51,4)$ & $20(54,1)$ & $17(45,9)$ & $20(54,1)$ & $17(45,9)$ \\
\hline Estase em valéculas & $10(27)$ & $27(73)$ & $20(54,1)$ & $17(45,9)$ & $23(62,2)$ & $14(37,8)$ & $18(48,6)$ & $19(51,4)$ \\
\hline Estase em recessos piriformes & $10(27)$ & $27(73)$ & $18(48,6)$ & $19(51,4)$ & $17(45,9)$ & $20(54,1)$ & $12(32,4)$ & $25(67,6)$ \\
\hline Penetração laríngea & $15(40,5)$ & $22(59,5)$ & $14(37,8)$ & $23(62,2)$ & $10(27)$ & $27(73)$ & $8(21,6)$ & $29(78,4)$ \\
\hline Aspiração traqueal & $6(16,2)$ & $31(83,8)$ & $3(8,1)$ & $34(91,9)$ & $3(8,1)$ & $34(91,9)$ & $1(2,7)$ & $36(97,3)$ \\
\hline
\end{tabular}

Legenda: $\mathrm{P}=$ número de pacientes com presença de manifestação disfágica para a determinada consistência; $\mathrm{A}=$ número de pacientes com ausência de manifestação disfágica para a determinada consistência

Teste estatístico comparativo entre os percentuais 
aumento das consistências alimentares ${ }^{(16)}$. Além disso, as variações de textura e quantidade de alimento oferecido estiveram associadas ao tempo do disparo da deglutição, à estase após deglutição e à aspiração ${ }^{(17)}$.

A presença de resíduos alimentares em valéculas e/ou recessos piriformes pode ocorrer devido às alterações na fase preparatória e/ou oral da deglutição, à ineficiência da ejeção do bolo alimentar, ao atraso no disparo do reflexo da deglutição, à diminuição dos movimentos peristálticos, à redução da elevação e anteriorização laríngea e/ou à incoordenação do músculo cricofaríngeo ${ }^{(18)}$.

Neste estudo, a estase em valéculas foi o achado mais observado, ocorrendo com maior frequência após a ingestão da consistência pastosa grossa, devido à falta de maior controle oral e de uma eficiência na propulsão da língua que seriam necessários para deglutir alimentos de maior viscosidade, em $62,1 \%$ (23/43), seguida pela pastosa fina em 54,1\% (20/43), sólida em 48,6\% (18/43) e líquida em 27,1\% (10/43). Em relação à estase em recessos piriformes, a consistência pastosa fina foi a mais frequente em 48,6\% (18/43), seguida pela pastosa grossa em 45,9\% (17/43), sólida em 32,4\% (12/43) e líquida em 27,1\% (10/43).

Estudos mostram que pacientes com comprometimento neurológico apresentam lentidão e falta de controle das estruturas, relacionadas às fases preparatória e oral da deglutição ${ }^{(19-24)}$, aumentando a quantidade de resíduos alimentares em valéculas e/ou recessos piriformes, o que acarreta maior risco para a penetração laríngea e/ou aspiração traqueal pós-deglutição ${ }^{(4)}$.

A presença de penetração laríngea apresentou maior incidência na administração da consistência líquida, em 40,5\% (15/43), seguida da pastosa fina em 37,8\% (14/43), pastosa grossa em 27\% (10/43) e sólida, em 21,6\% (8/43).

A aspiração traqueal esteve presente para a consistência líquida em 16,2\% (6/43), seguida da pastosa fina e pastosa grossa em 8,1\% (3/43) e sólida em 2,7\% (1/43).

A penetração laríngea e a aspiração traqueal podem acontecer antes, durante e/ou após a deglutição ${ }^{(23)}$. Todavia, em virtude da limitação da VED para analisar a fase faríngea da deglutição, os achados foram classificados apenas quanto à sua ocorrência antes ou após a deglutição. Em relação às consistências: líquida e pastosa fina, as manifestações foram observadas antes do início da deglutição; quanto às consistências pastosa grossa e sólida, ocorreram após a deglutição.

Estudos mostram que, para a ingestão de líquidos, é necessária uma deglutição elaborada, pela necessidade de controle do fluxo descoordenado e rápido escoamento da consistência, o que exige um maior controle respiratório e muscular para impedir a invasão da via aérea pelo alimento ${ }^{(25-27)}$.

\section{CONCLUSÃO}

A videoendoscopia da deglutição em pacientes com disfagia orofaríngea neurogênica permitiu uma avaliação morfofuncional da fase faríngea da deglutição, onde se observou a interrelação existente entre as fases da deglutição.

No presente estudo, os comprometimentos relacionados à fase oral da deglutição foram os de maior incidência no grupo de pacientes, o que influenciou os achados do exame relacionados à fase faríngea da deglutição.

As consistências de maior textura foram as que causaram a maior porcentagem de manifestações disfágicas durante o exame, como a estase em valéculas e o atraso no disparo do reflexo da deglutição.

Apesar de ser uma análise da deglutição apenas no momento sob teste e não possibilitar a visualização da fase faríngea da deglutição, a videoendoscopia da deglutição mostrou-se um instrumento eficaz para a caracterização das manifestações disfágicas na população estudada.

\begin{abstract}
Purpose: To describe the main findings of fiberoptic endoscopy of swallowing in adults with neurogenic oropharyngeal dysphagia. Methods: A retrospective study was carried out using 46 medical records of patients with diagnosis of neurogenic oropharyngeal dysphagia, assisted in the period from July 2004 to December 2007 at the Dysphagia Ambulatory of the University of Fortaleza. The average age of the patients was 58.9 years, ranging between 18 and 85 years; $51.1 \%$ (22/43) were male and 48.9\% (22/46) female. The exams were considered altered in the presence of one or more of the following dysphagic manifestations: premature spillage, delayed swallowing, residues in vallecules and/or pyriform recess, laryngeal penetration and/or tracheal aspiration. Results: The main findings observed, in descending occurrence order, were: presence of residues in vallecules for pasty thick consistency, delay in swallowing during pasty thick and solid consistencies ingestion, presence of residues in pyriform recesses after the swallowing of pasty fine consistency, premature spillage for liquids, laryngeal penetration and tracheal aspiration for liquids before swallowing. Conclusion: The main dysphagic manifestations observed were related to alterations in the volunteer phases of deglutition and to the increase of the food consistencies. The fiberoptic endoscopy of swallowing proved to be an effective tool for the diagnosis of oropharyngeal dysphagias.
\end{abstract}

Keywords: Deglutition; Dysphagia; Endoscopy/methods; Endoscopy/utilization; Deglutition disorders 


\section{REFERÊNCIAS}

1. Bass NH, Morrell RM. The neurology of swallowing. In: Groher ME. Dysphagia: diagnosis and management. 2nd ed. Boston: ButterworthHeinemann; 1992. p.1-29.

2. Furkim AM, Silva RG. Conceitos e implicações para a prática clínica e para a classificação da disfagia orofaríngea neurogênica. In: Furkim AM, Silva RG. Programas de reabilitação em disfagia neurogênica. São Paulo: Frôntis; 1999. p. 1-20.

3. Junqueira P. A importância da fase oral na dinâmica da deglutição. In: Costa MM, Castro LP. Tópicos em deglutição e disfagia. Rio de Janeiro: Medsi; 2003. p.31-6.

4. Costa MM, Nova JL, Carlos MT, Pereira AA, Koch HA. Videofluoroscopia: um novo método. Radiol Bras. 1992;25(1):11-8.

5. Hafner G, Neuhuber A, Hirtenfelder S, Schmedler B, Eckel HE. Fiberoptic endoscopic evaluation of swallowing in intensive care unit patients. Eur Arch Otorhinolaryngol. 2008; 265(4):441-6.

6. Doggett DL, Tappe KA, Mitchell MD, Chapell R, Coates V, Turkelson $\mathrm{CM}$. Prevention of pneumonia in elderly stroke patients by systematic diagnosis and treatment of dysphagia: an evidence-based comprehensive analysis of the literature. Dysphagia. 2001;16(4):279-95.

7. Ekberg O, Hamdy S, Woisard V, Wuttge-Hanning A, Ortega P. Social and psychological burden of dysphagia: its impact on diagnosis and treatment. Dysphagia. 2002;17(2):139-46.

8. Chen PH, Golub JS, Hapner ER, Johns MM 3rd. Prevalence of perceived dysphagia and quality-of-life impairment in a geriatric population. Dysphagia. 2009;24(1):1-6.

9. Wakasugi Y, Tohara H, Hattori F, Motohashi Y, Nakane A, Goto S et al. Screening test for silent aspiration at the bedside. Dysphagia. 2008;23(4):364-70.

10. Seidl RO, Nusser-Müller-Busch R, Westhofen M, Ernst A. Oropharyngeal findings of endoscopic examination in swallowing disorders of neurological origin. Eur Arch Otorhinolaryngol. 2008;265(8):963-70.

11. Aviv JE, Murry T, Zschommler A, Cohen M, Gartner C. Flexible endoscopic evaluation of swallowing with sensory testing: patient characteristics and analysis of safety in 1,340 consecutive examinations. Ann Otol Rhinol Laryngol. 2005;114(3):173-6.

12. Langmore SE. Evaluation of oropharyngeal dysphagia: which diagnostic tool is superior? Curr Opin Otolaryngol Head Neck Surg. 2003;11(6):485-9.

13. Flaksman H, Ron Y, Ben-David N, Cinamon U, Levy D, Russo E et al. Modified endoscopic swallowing test for improved diagnosis and prevention of aspiration. Eur Arch Otorhinolaryngol. 2006;263(7):63740.

14. Eisenhuber E, Schima W, Schober E, Pokieser P, Stadler A, Scharitzer $\mathrm{M}$ et al. Videofluoroscopic assessment of patients with dysphagia: pharyngeal retention is a predictive factor for aspiration. AJR Am J Roentgenol. 2002;178(2):393-8.

15. Langmore SE, Schatz K, Olson N. Fiberoptic endoscopic examination of swallowing safety: a new procedure. Dysphagia. 1988;2(4):216-9.

16. Daniels SK, Corey DM, Hadskey LD, Legendre C, Priestly DH, Rosenbek JC et al. Mechanism of sequential swallowing during straw drinking in healthy young and older adults. J Speech Lang Hear Res. 2004;47(1):33-45.

17. Rogers B, Arvedson J, Buck G, Smart P, Msall M. Characteristics of dysphagia in children with cerebral palsy. Dysphagia. 1994;9(1):69-73.

18. Costa MM, Moscovici M, Pereira AA, Koch HA. A avaliação videofluoroscópica da transição faringoesofágica (esfíncter superior do esôfago). Radiol Bras. 1993;26(2):71-80.

19. Ding R, Logemann JA. Pneumonia in stroke pacients: a retrospective study. Dysphagia. 2000;15(2):51-7.

20. Jafari S, Prince RA, Kim DY, Paydarfar D. Sensory regulation of swallowing and airway protection: a role for the internal superior laryngeal nerve in humans. J Physiol. 2003;550(Pt 1):287-304.

21. Pikus L, Levine MS, Yang YX, Rubesin SE, Katzka DA, Laufer I, Gefter WB. Videofluoroscopic studies of swallowing dysfunction and the relative risk of pneumonia. AJR Am J Roentgenol. 2003;180(6):1613-6.

22. Myssiorek D. Recurrent laryngeal nerve paralysis: anatomy and etiology. Otolaryngol Clin North Am. 2004;37(1):25-44.

23. Sulica L. The superior laryngeal nerve: function and dysfunction. Otolaryngol Clin North Am. 2004;37(1):183-201.

24. Power ML, Hamdy S, Singh S, Tyrrell PJ, Turnbull I, Thompson DG. Deglutitive laryngeal closure in stroke patients. J Neurol Neurosurg Psychiatry. 2007;78(2):141-6.

25. Marik PE. Aspiration pneumonitis and aspiration pneumonia. N Engl J Med. 2001;344(9):665-71.

26. Vergis EN, Brennen C, Wagener M, Muder RR. Pneumonia in longterm care: a prospective case-control study of risk factors and impact on survival. Arch Intern Med. 2001;161(19):2378-81.

27. Lazarus CL, Logemann JA, Radermaker AW, Kahrilas PJ, Pajak T, Lazar R et al. Effects of bolus volume, viscosity, and repeated swallows in nonstroke subjects and stroke patients. Arch Phys Med Rehabil. 1993;74(10):1066-70.

Anexo 1. Protocolo de avaliação fonoaudiológica

I- Identificação

Nome:

Gênero:

Diagnóstico médico:

Responsável:

Endereço:

II- História clínica

Queixa:

Evolução da doença:

Comprometimento da linguagem expressiva

Comprometimento da linguagem receptiva

Comprometimento de fala

Déficit cognitivo

Déficit de atenção

Perda de peso

Broncopneumonias

Independência alimentar
( ) $\operatorname{Sim}$ ( ) Não
( ) Sim ( ) Não
( ) $\operatorname{Sim}$ ( ) Não
( ) Sim ( ) Não
( ) Sim ( ) Não
( ) $\operatorname{Sim}$ ( ) Não
( ) Sim ( ) Não

( ) Parcial ( ) Total ( ) Ausente 
Condição respiratória:

$\begin{array}{lll}\text { Ventilação mecânica } & \text { ( ) Sim } & \text { ( ) Não Tempo? } \\ \text { Intubação } & \text { ( ) Sim ( ) Não } & \\ \text { Extubação } & \text { ( ) Sim ( ) Não Tempo? } \\ \text { Traqueostomia } & \text { ( ) Sim ( ) Não } & \\ & \text { ( ) Cânula de silicone } & \\ \text { SpO2 } & \text { ( ) Cânula sem cuff } & \text { ( ) Cânula com cuff } \\ & \text { ( ) Sim ( ) Não }\end{array}$

Alimentação atual:

Via de alimentação

( ) Via enteral Volume por refeição:

$\begin{array}{lll}\text { ( ) SNG ( ) SNE ( ) GST ( ) outra: } & \\ \text { Dieta } & \text { ( ) industrializada } & \text { ( ) caseira }\end{array}$

Intercorrências
( ) tosse/engasgo
( ) regurgitamento
( ) redução I vocal
( ) alteração qualidade vocal
( ) alteração respiratória
( ) cianose
( ) asfixia / sufocamento
( ) ausentes

( ) outras:

Manobras compensatórias:

( ) Via oral

Volume por refeição:

Consistências:

Sabores e temperatura preferidos:

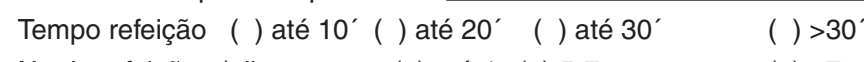

$\begin{array}{llll}\text { No de refeições / dia } & (\text { ) até } 4 & \text { ( ) } 5-7 & (\text { ) }>7\end{array}$

Postura usual para alimentação
( ) sentado
( ) semi-deitado
( ) deitado

Utensílios:

Intercorrências

$\begin{array}{lll}\text { ( ) tosse/engasgo } & (\text { ) regurgitamento } & (\text { ) redução I vocal } \\ \text { ( ) alteração qualidade vocal } & (\text { ) alteração respiratória }\end{array}$

( ) cianose ( ) asfixia / sufocamento ( ) ausentes

( ) outras:

Manobras compensatórias:

III- Avaliação fonoaudiológica

Achados motores globais

\section{Equilíbrio}

controle cervical

controle de tronco
( ) parcial
( ) total
( ) ausente
( ) senta c/ apoio
( ) senta s/ apoio
( ) ausente

Achados estruturais e motores orais
Prótese
( ) sim:
( ) não
Tipologia facial
( ) dólico
( ) mésio
( ) braqui
Oclusão
( ) Classe I
( ) Classe II
( ) Classe II - div. I
( ) Classe II - div. II
( ) Classe III

Tônus motor-oral

$\begin{array}{llll}\text { Lábios } & \text { ( ) hipertônico ( ) hipotônico ( ) flutuante ( ) normal } \\ \text { Língua } & \text { ( ) hipertônico ( ) hipotônico ( ) flutuante ( ) normal } \\ \text { Bochechas } & \text { ( ) hipertônico ( ) hipotônico ( ) flutuante ( ) normal } \\ \text { Mento } & \text { ( ) hipertônico ( ) hipotônico ( ) flutuante ( ) normal }\end{array}$

Sensibilidade

$\begin{array}{llll}\text { Intra-oral } & (\text { ) normal } & (\text { ) reduzida } & \text { ( ) exacerbada } \\ \text { Extra-oral } & (\text { ) normal } & (\text { ) reduzida } & \text { ( ) exacerbada }\end{array}$

Mobilidade

$\begin{array}{llll}\text { Lábios } & \text { ( ) normal } & \text { ( ) reduzida } & \text { ( ) exacerbada } \\ \text { Bochechas } & \text { ( ) normal } & \text { ( ) reduzida } & \text { ( ) exacerbada } \\ \text { Mandíbula } & \text { ( ) normal } & \text { ( ) reduzida } & \text { ( ) exacerbada } \\ \text { Língua } & \text { ( ) normal } & \text { ( ) reduzida } & \text { ( ) exacerbada } \\ \text { Palato mole } & \text { ( ) normal } & \text { ( ) reduzida } & \text { ( ) exacerbada }\end{array}$


Morfologia

$\begin{array}{lll}\text { Lábios } & \text { ( ) normal } & \text { ( ) alterada: } \\ \text { Bochechas } & \text { ( ) normal } & \text { ( ) alterada: } \\ \text { Mandíbula } & \text { ( ) normal } & \text { ( ) alterada: } \\ \text { Língua } & \text { ( ) normal } & \text { ( ) alterada: } \\ \text { Palato mole } & \text { ( ) normal } & \text { ( ) alterada: } \\ \text { Assoalho } & \text { ( ) normal } & \text { ( ) alterada: }\end{array}$

Avaliação funcional da alimentação

Mastigação
Padrão
( ) unilateral
( ) bilateral
( ) vertical

Formação e controle do bolo alimentar
( ) amassa o alimento
( ) tritura o alimento
( ) movs. rotacionais de mandb.
( ) movs. verticais de mandb.
( ) lateralização de língua
( ) anteriorização de língua
( ) movs. de lg ausentes
( ) escape anterior de alimentos

Deglutição

Líquido - volume:

( ) escape anterio

( ) trânsito oral aumentado

( ) tosse/engasgo antes deglutição

( ) tosse/engasgo durante deglutição

( ) tosse/engasgo após deglutição

( ) redução elevação LA

( ) voz molhada após deglutição

( ) deglutições múltiplas

( ) alteração respiratória

( ) regurgitamento

( ) ausculta cervical +

( ) sem intercorrências

Manobras efetivas:

Pastoso Fino - volume:
( ) escape anterior
( ) trânsito oral aumentado
( ) tosse/engasgo antes deglutição
( ) tosse/engasgo durante deglutição
( ) tosse/engasgo após deglutição
( ) redução elevação LA
( ) voz molhada após deglutição
( ) deglutições múltiplas
( ) alteração respiratória
( ) regurgitamento
( ) ausculta cervical +
( ) sem intercorrências

Manobras efetivas:

Pastoso Grosso - volume:

( ) escape anterior

( ) trânsito oral aumentado

( ) tosse/engasgo antes deglutição

( ) tosse/engasgo durante deglutição

( ) tosse/engasgo após deglutição

( ) redução elevação LA

( ) voz molhada após deglutição

( ) deglutições múltiplas

( ) alteração respiratória

( ) regurgitamento

( ) ausculta cervical +

( ) sem intercorrências

Manobras efetivas:

Sólido - volume:
( ) escape anterior
( ) trânsito oral aumentado
( ) tosse/engasgo antes deglutição
( ) tosse/engasgo durante deglutição
( ) tosse/engasgo após deglutição
( ) redução elevação LA
( ) voz molhada após deglutição
( ) deglutições múltiplas
( ) alteração respiratória
( ) regurgitamento
( ) ausculta cervical +
( ) sem intercorrências

Manobras efetivas:

Diagnóstico fonoaudiológico:

Condutas e encaminhamentos: 
Anexo 2. Protocolo de avaliação videoendoscópica da deglutição

Nome:

D.N.: Idade atual: Data:

I - Avaliação endoscópica anatômica e funcional

Observações anatômicas

Língua:

Véu palatino:

Faringe:

Laringe:

Observações funcionais

$\begin{array}{lcc}\text { Mobilidade de lábios } & (\text { ) adequada } & (\text { ) inadequada } \\ \text { Vedamento labial } & (\text { ) adequado } & (\text { ) inadequado } \\ \text { Mobilidade de língua } & (\text { ) adequada } & (\text { ) inadequada } \\ \text { Sensibilidade de língua } & (\text { ) adequada } & (\text { ) inadequada } \\ \text { Mobilidade do véu palatino } & (\text { ) adequada } & (\text { ) inadequada } \\ \text { Fechamento velopalatino } & \\ (\text { ) fechamento velofaríngeo } & (\text { ) desvios de eixo } \\ (\text { ) escape de ar } & (\text { ) sinal de passavant }\end{array}$

Faringe

( ) estase salivar / resíduos alimentares em valéculas

( ) estase salivar / resíduos alimentares em seios piriformes

II - Estudo de deglutição

Consistência líquida

Retenção do bolo na cavidade oral Início da deglutição após o comando Trânsito orofaríngeo

Regurgitação nasal

Estase em valéculas

Estase em recessos piriformes

Penetração

$$
\text { ( ) face laríngea epiglote }
$$

( ) espaço interaritenóideo

Aspiração laringotraqueal
( ) face laríngea epiglote
( ) espaço interaritenóideo

Reflexo de tosse

Voz úmida

Consistência pastosa fina

Retenção do bolo na cavidade oral Início da deglutição após o comando

Trânsito orofaríngeo

Regurgitação nasal

Estase em valéculas

Estase em recessos piriformes

Penetração

( ) face laríngea epiglote

( ) espaço interaritenóideo

Aspiração laringotraqueal
( ) face laríngea epiglote
( ) espaço interaritenóideo

Reflexo de tosse

Voz úmida
( ) eficiente
( ) ineficiente
( ) eficiente
( ) retardado
( ) eficiente
( ) retardado
( ) presente
( ) ausente
( ) presente
( ) ausente
( ) presente
( ) ausente
( ) ligamentos ariepiglóticos
( ) ausente
( ) ligamentos ariepiglóticos
( ) ausente
( ) presente
( ) ausente
( ) presente
( ) ausente
( ) eficiente
( ) ineficiente
( ) eficiente
( ) retardado
( ) eficiente
( ) retardado
( ) presente
( ) ausente
( ) presente
( ) ausente
( ) presente
( ) ausente
( ) ligamentos ariepiglóticos
( ) ausente
( ) ligamentos ariepiglóticos
( ) ausente
( ) presente
( ) ausente
( ) presente
( ) ausente 
Consistência pastosa grossa

Retenção do bolo na cavidade oral Início da deglutição após o comando
( ) eficiente
( ) ineficiente
( ) eficiente
( ) retardado
( ) eficiente
( ) retardado
( ) presente
( ) ausente
( ) presente
( ) ausente
( ) presente
( ) ausente

Trânsito orofaríngeo

Regurgitação nasal

Estase em valéculas

Estase em recessos piriformes

Penetração

( ) face laríngea epiglote

( ) ligamentos ariepiglóticos

( ) espaço interaritenóideo

( ) ausente

Aspiração laringotraqueal

( ) ligamentos ariepiglóticos

( ) face laríngea epiglote

( ) espaço interaritenóideo

Reflexo de tosse

( ) ausente

( ) presente ( ) ausente

Voz úmida

( ) presente

( ) ausente

Consistência sólida

Retenção do bolo na cavidade oral

Início da deglutição após o comando

Trânsito orofaríngeo

Regurgitação nasal

Estase em valéculas

Estase em recessos piriformes

Penetração

( ) face laríngea epiglote

( ) espaço interaritenóideo

( ) eficiente ( ) ineficiente

( ) eficiente ( ) retardado

( ) eficiente ( ) retardado

( ) presente ( ) ausente

( ) presente ( ) ausente

( ) presente ( ) ausente

( ) ligamentos ariepiglóticos

( ) ausente

Aspiração laringotraqueal

( ) face laríngea epiglote

( ) espaço interaritenóideo

( ) ligamentos ariepiglóticos

( ) ausente

( ) presente ( ) ausente

Reflexo de tosse

( ) presente

( ) ausente

III - Avaliação da sensibilidade laríngea

Reflexo de adução glótica

( ) presente

( ) ausente

IV - Conclusão e orientação

Consistências indicadas:

Volume indicado:

Manobras efetivas:

Encaminhamentos:

Sugestões:

Conclusão:

Data:

Médico responsável:

Fonoaudiólogo responsável: 